\title{
Closing open bite by chewing on the posterior teeth
}

\author{
C. Ben Younes-Uzan
}

Qualified Specialist Dento-Facial Orthopedics, Former consulting assistant at Robert Debré hospital SUMMARY

A device on the upper arch made from resin in order to disengage the teeth and practice an altered unilateral chewing allowing the stimulation of the three dimensional growth of the maxilla, and the repositioning of the mandible.

This article will show the early resolution of the open bite and the anterior vertical excess.

KEYWORDS

Chewing, lateral movements, posterior elevations, open bite

\section{INTRODUCTION}

Open bites are caused and maintained by the persistence of sucking habits, suppression of the cause associated with reeducation may be a therapeutic option, but masticatory stimulation by putting all of the teeth back into function also provides lasting results.

\section{PRINCIPLES OF TREATMENT}

Chewing is the main function of the teeth. Professor Planas in his book Neuro-Occlusal Rehabilitation showed that chewing stimuli gave energy to the growth and development of the alveolo-dental arches ${ }^{8}$.

The primitive populations after breast feeding, without bottle or nipple, have a hard, dry, solid chew; in these societies where the teeth are physiologically abraded, there are no periodontal problems and almost no need for orthodontics. The baby's feeding begins with a long period of breastfeeding, causing the mandible to symmetrically move forward and prohibiting oral breathing. As the temporary

Address for correspondence:

Carine Ben Younes-Uzan

60, Cours de Vincennes - 75012 Paris

E-mail: c.benyounes@wanadoo.fr

This is an Open Access article distributed under the terms of the Creative Commons Attribution License (http://creativecommons.org/licenses/by/4.0), which permits unrestricted use, distribution, and reproduction in any medium, provided the original work is properly cited. 
dentition is established, alternating unilateral chewing takes place, causing all teeth to rub except the canine swinging alternately to the right and to the left ${ }^{8}$.

In infants, the tongue is spread out between the alveolar arches and, in the absence of suction persistence, not to be "eaten", the dental friction will cause its recoil ${ }^{12}$.

There is a real antagonism between the tongue and the teeth, and two therapeutic approaches: to roll back the tongue or to make the teeth work.

The physiological stimulation of the dental organs leads to their occlusal and proximal migration and a micro-depression, it is compensated by a physiological, occlusal and inter-proximal microattrition ${ }^{8}$.

The wearing of a system that allows all the maxillary teeth, including those articulated cross or open bite receive stimulation, and allowing the expression of the vertical response as required, will allow the correction of the vertical direction.

The mandible consists of two embryological buds and the maxilla of three buds, Planas found that the excitation of a tooth bud gave the answer to all of its teeth.

Just as a retro-incisal plane allows the opening in the vertical direction, planes molar elevations ${ }^{4}$ generating chewing on the posterior teeth will lead to closure of the vertical direction.

The lateral movements give a stimulus to all the teeth ${ }^{8}$ and the interposition of resin prohibits the peeling response where it covers the teeth, so the molar elevations will allow the closure of the mandibular angle with the egression of the anterior-inferior teeth. and some posterior intrusion.

The action is all the more rapid and simple when the patient is young ${ }^{5}$.

Grinding of the lateral temporary teeth, from the canine to the milk molar, can amplify the action of the apparatus at the same time as the plans will be adjusted to increase their ingressive action specifically on the 6-year-old teeth. Grinding to reduce DVO is to be avoided in class III cases where the maintenance of an occlusion height is essential for the durability of anteroposterior results ${ }^{1}$.

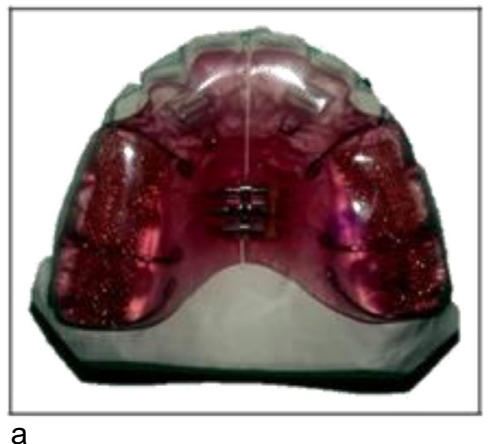

b

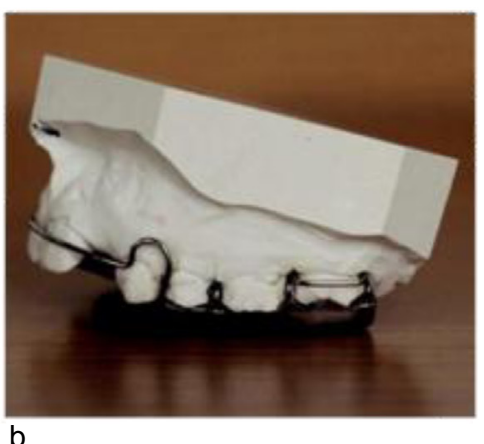

Figure 1 a through $c$. 


\section{THE DEVICE}

The device is a Hawley plate bearing molar elevations according to Def$f e z^{1,3,6,7}$, disengaging the occlusion and allowing mastication on both sides. Since the resin is in contact with all the maxillary teeth, the stimuli from laterality movements are transmitted to all the teeth in the arch, including those that were crossarticulated or the open bite before wearing the device.

If the incisors are to be retracted, a vestibular arch is added (Fig. 1a, b), in

\section{Case No. 1}

In this patient aged 8 years and 10 months, the open bite is closed in less than one semester (Fig. 2a, b). An extremely lightweight elastic (2 oz 8B) was placed on two buttons glued on 74 and 84 to retract the lower incisors and

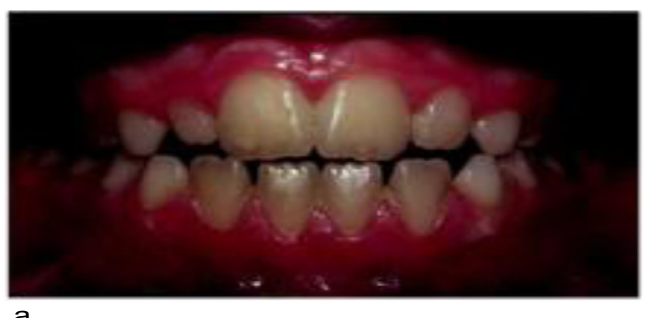

a

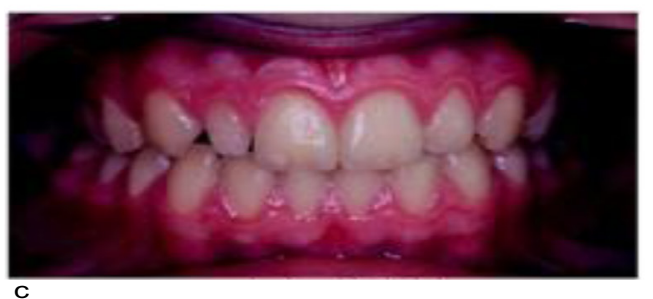

Class III cases, a retro-incisal arc or anterior cylinder is used (Fig. 1c).

The posterior elevations are smooth, they stop before the canines, their height will be greater than the free space of open bite; in case of articulated cross, the height will prevent the effect of premature contact which leads to mandibular deviation.

Cylinders and springs can reactivate transverse stimulation and add more localized actions, which is sometimes necessary on permanent teeth. to prevent them from colliding with the superior ones end to end (Fig. 2b).

The patient is reviewed in definitive dentition (Fig. 2c) to retract the maxillary lateral teeth in order to put them in class I and to increase the mesio-distal diameter of 12 size (Fig. $2 d$ ).

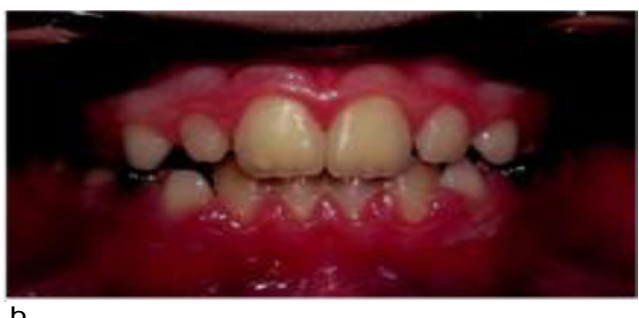

b

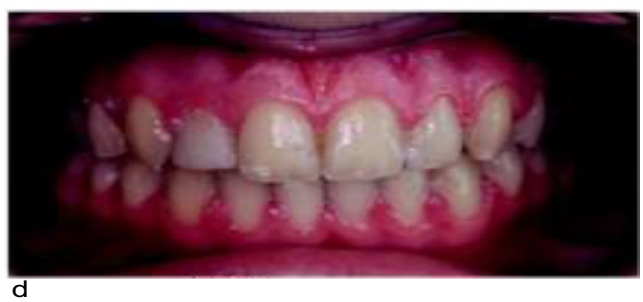

Figure 2

a through d. 
The incisal recovery obtained quickly was maintained between the stopping of the functional phase and the

Case No. 2

The patient presents a significant open bite on a hyperdivergent facial pattern, she is paired Age 6 years 4 months (Fig. 3a) using a plate

Molar elevations, we obtain a closing primer of the open bite, after 9 months the device is removed. (Figure $3 b$ and $c$ ).

The patient is seen after the breakthrough of the lateral incisors, the lack of recovery remained identical to that obtained at the removal of the device (Fig. 3d). An over-elevation treatment multi-fastening recovery without the need for restraint, the chewing function acting as a natural stabilization. phase, combined with an anterior elastic with a very light force, is repositioned, so that the mandibular incisors recede and pass behind the upper ones (Fig. 3e) and the open bite continues to close (Fig. $3 f$ and g).

The treatment is finished in self-ligating passive multi-attachment (Fig. 3h before treatment, Fig. 3i after treatment).

The first teleradiography is performed before the functional treatment (Fig. 4a), the second before the
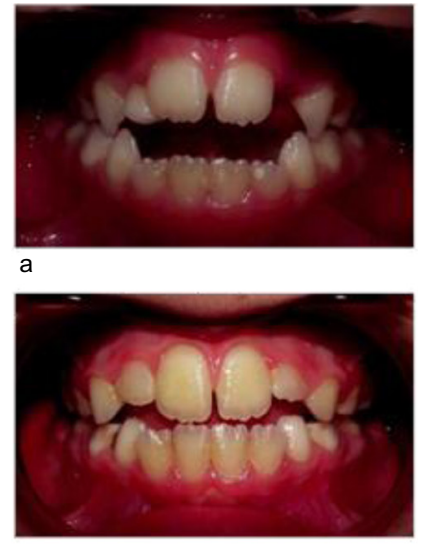

d

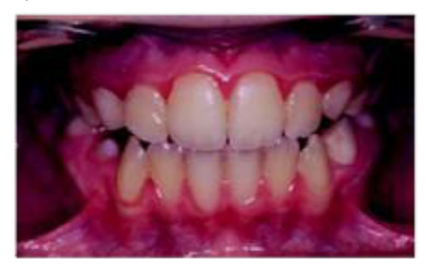

g

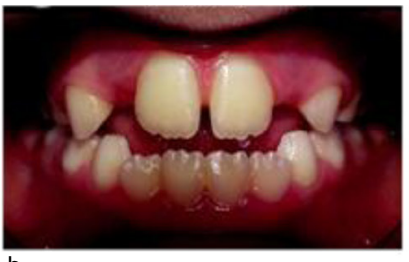

b

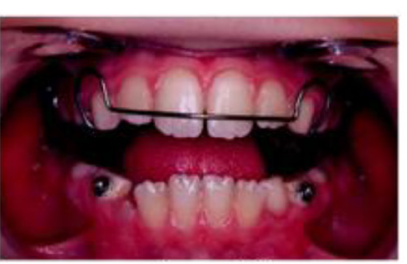

e
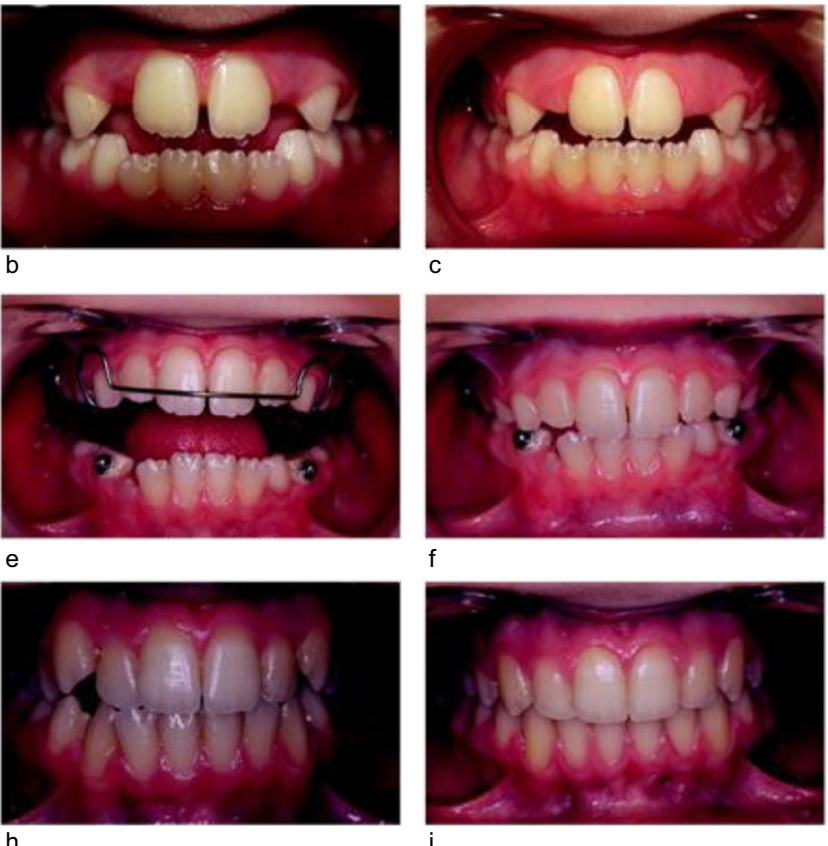

c

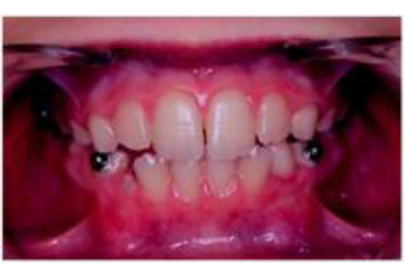

$f$

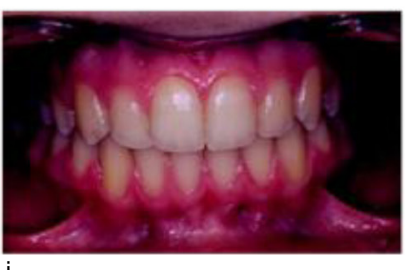

Figure 3

a through $\mathrm{c}$

$d$ through $f$

$g$ through i. 

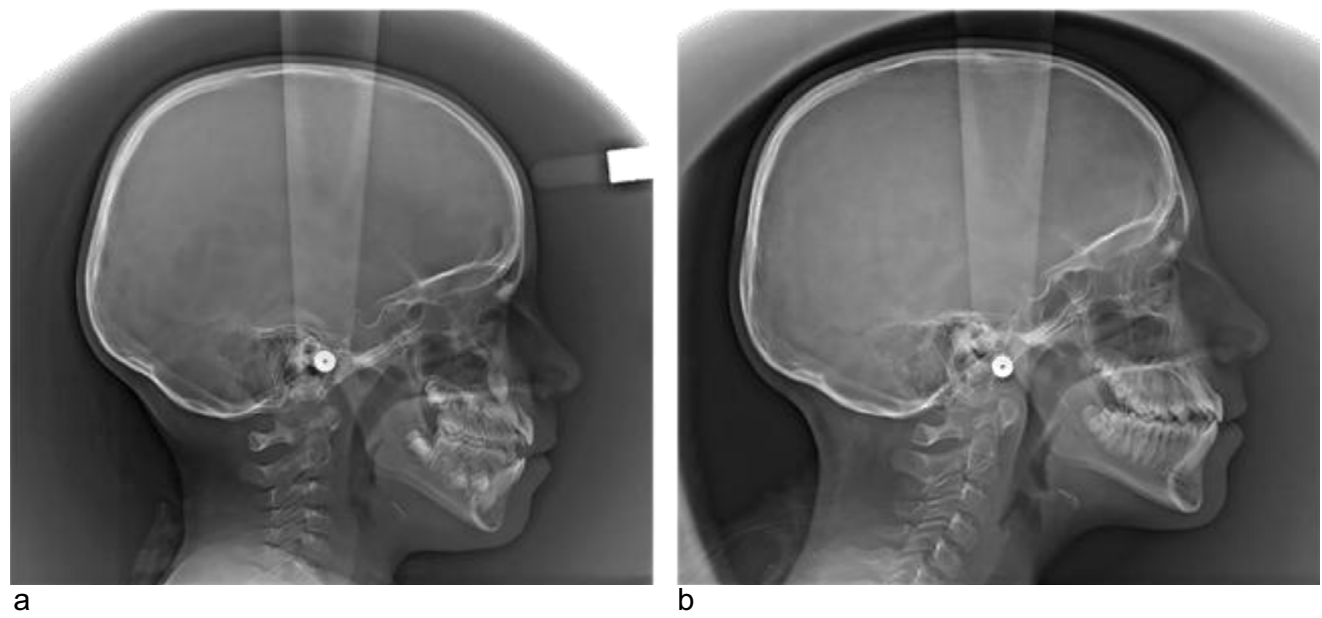

Figure 4

a through $b$.

multi-attachment finishing (Fig 4b), we observe a closure of the mandibular angle, the skeletal pattern at the intital hyperdivergent has evolved positively by becoming normalized.
This modification is possible because the treatment is performed early at an age when there is still a lot of growth to come, it allows to replace the skeletal evolution in a virtuous circle.

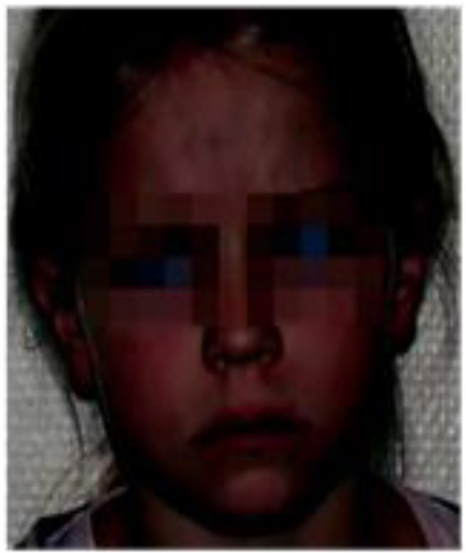

a

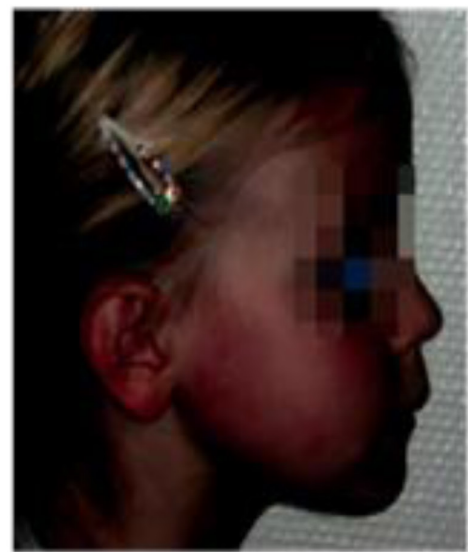

b

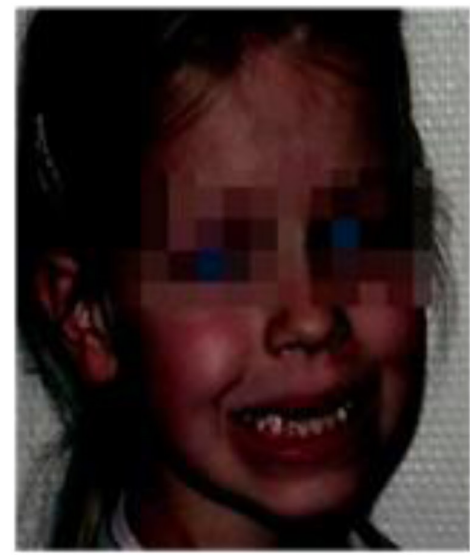

C

Figure 5

a through $c$. 

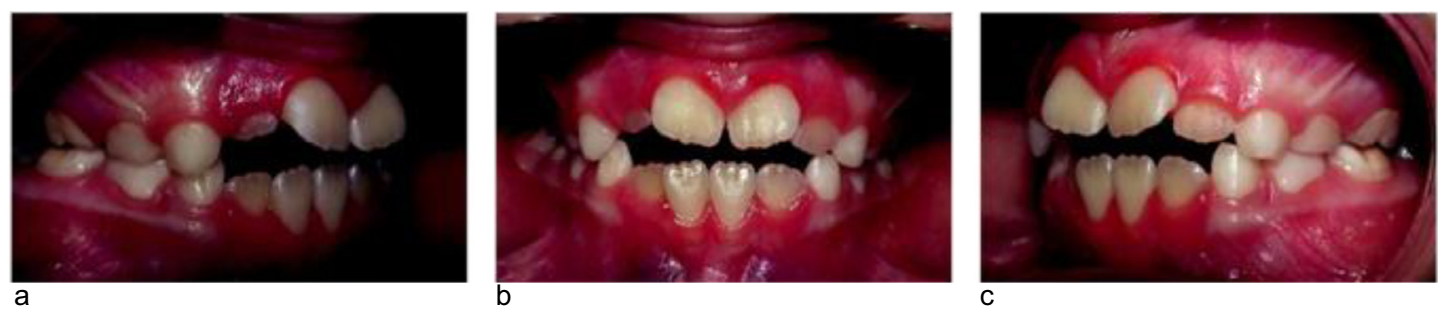

Figure 6

a through $c$.
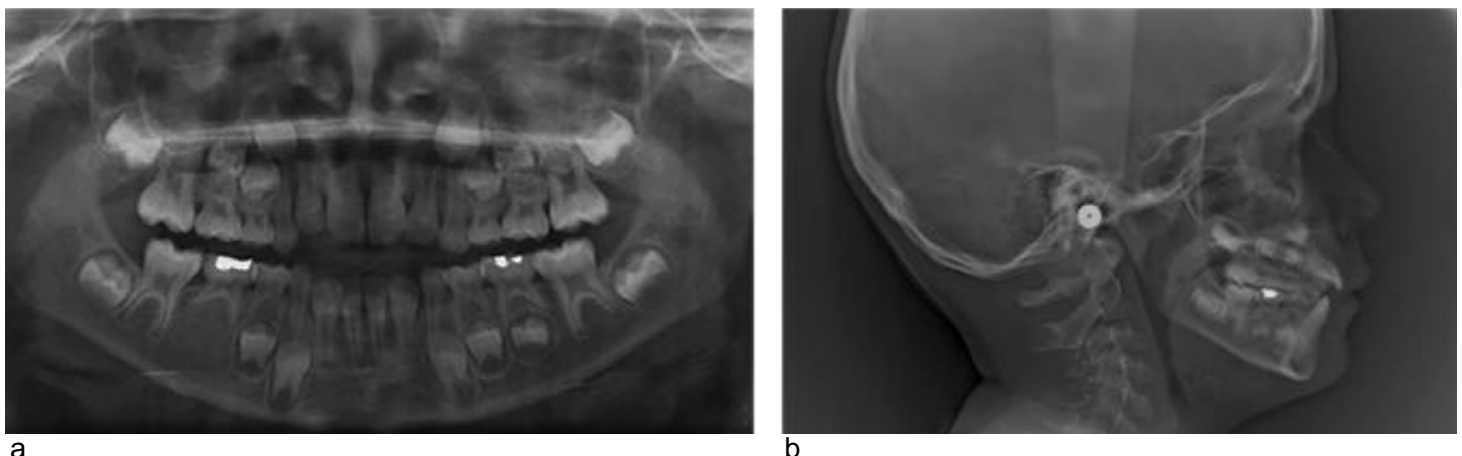

Figure 7

a through $b$.
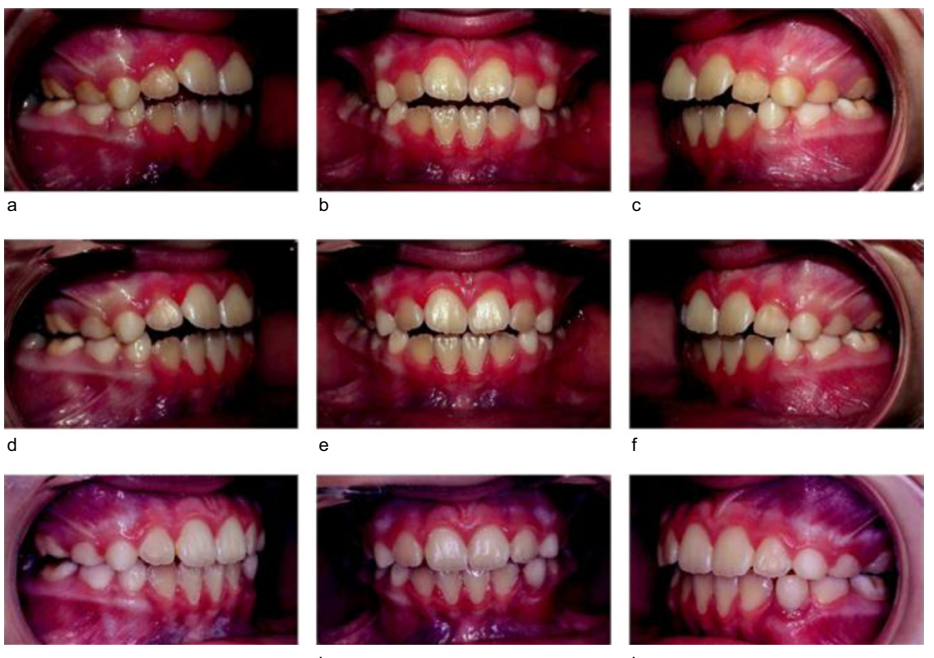

Figure 8

a through $i$. 


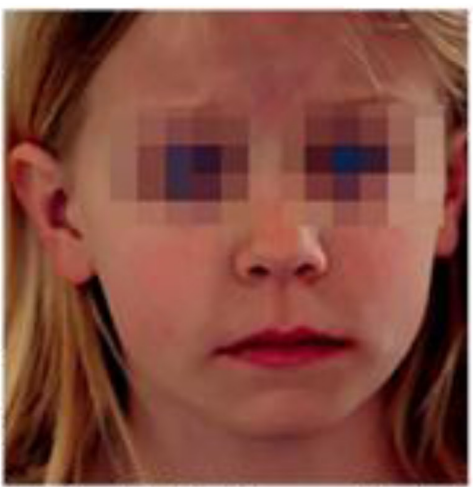

a

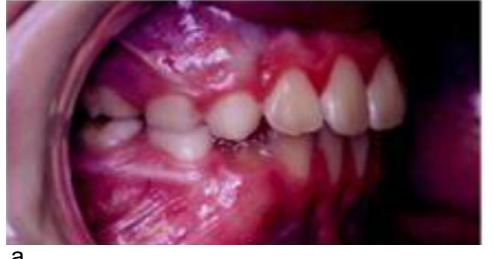

a

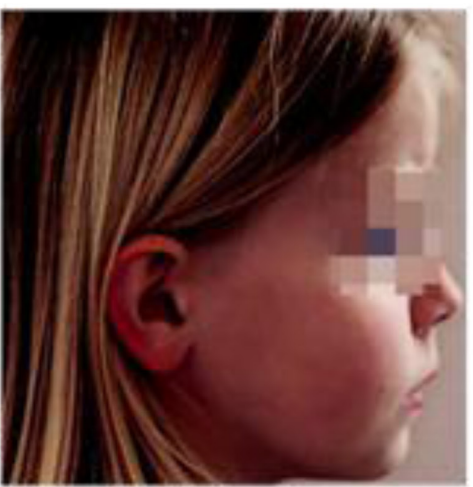

b

Figure 9

a through $c$.

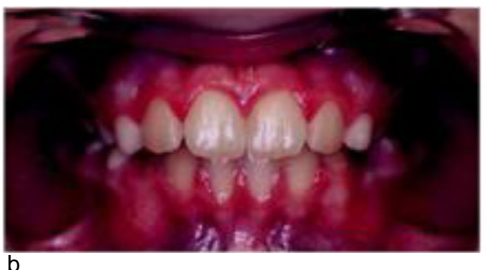

Figure 10

a through $\mathrm{c}$.

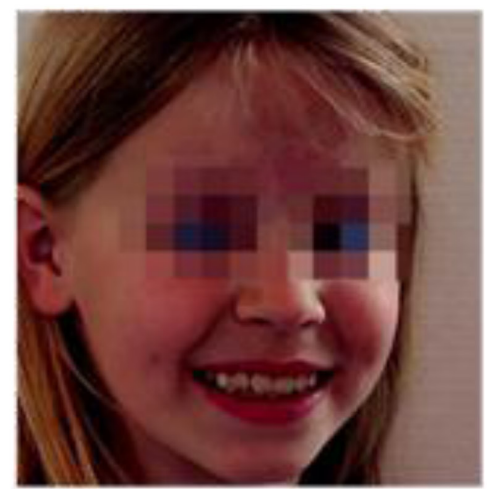

C

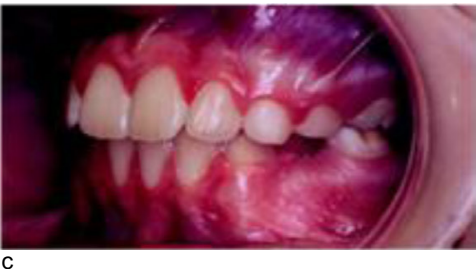

c

\begin{abstract}
(1)
\end{abstract}




\section{CASE No. 3}

This 7-year-old, 2-month-old patient (Fig. 5) has asthma, and has been thumb sucking for a long time. She has a maxillary endognathia with 16 articulated cross and thus a mandibular shift to the right giving a class I on the left and a small class II on the right. Open bite concerns the incisal area (Fig. 6, a to c), the upper incisors are in proalveolina (Fig. 7b).

On the panoramic radiograph, there is the agenesis of 45 and the current absence of the 8 (Fig. 7a), the malocclusion generates a lack of space in the upper arch the perimeter of which is reduced. This encumbrance is visible clinically in the anterior zone and laterally on the X-ray.

The treatment is carried out using a plate provided with molar elevations to close the vertical direction.

\section{CONCLUSION}

The lack of therapeutic management of the open bite will lead to the development of significant increases in vertical direction and skeletal hyperdivergences. The early treatment of anterior open bite by chewing on the posterior teeth with removable plates with molar elevations, which are little "removable
A cylinder placed most posteriorly allows the expansion of the arch and the resolution of the articulated cross by putting the 16 in normocclusion. The vestibular arch recedes and aligns the upper incisors, while the expansion increases the arch perimeter, giving room for the incisors (Fig. 8a-i).

The patient is reviewed with a radiological assessment (Fig. 9, 10, 11): the smile is better centered and the occlusion is normalized. She benefited from twice 6 months of the device with a break between the two times.

The nasal breathing is improved: the widening of the ceiling of the mouth corresponding to the increase of the area of the nasal fossae.

direct tracks", makes it possible to normalize the skeletal relationships, to reorient the maxillary and mandibular growth to obtain an inter-arch harmony that will continue with future growth.

Conflict of interest: The author declares that there is no conflict of interest.

\section{BIBLIOGRAPHY}

1. Ben Younes-Uzan C. proposed unconventional treatment of class III. French orthodontics 2007;82(2):133.

2. Couly G. The tongue, natural device of dento facial orthopedics for better and for worse. Rev Orthop Dento-Faciale 1989;23:9-17. 
3. Deffez JP. Prognathies mandibular. Therapeutic proposals. Paris: Julien Prelat, 1971.

4. Deffez JP. Bases of the middle floor of the face. Sites and growth factors of the maxillary: current data. Odonto-Stomatol Act 1979;128:647-668.

5. Davis J. architecturally and Craniofacial dynamics. Pediatric stomato-logie postgraduate courses. Hospital Robert Debré, 1992-1993.

6. Fellus P. Early orthodontics in deciduous dentition. Paris: CDP, 2003.

7. Pepper M, Garlopeau F, Laudenbach P. Prognathism mandibular treated by deciduous elevation of the articulated. Act Odonto-Stomatol 1959;47:277-290.

8. Planas P. The Neuro-occlusal Rehabilitation. Paris, Masson, 1992. 